\title{
Plasmonic quantum cascade laser antenna
}

\section{Citation}

Yu, Nanfang, Ertugrul Cubukcu, Laurent Diehl, Mikhail A. Belkin, Kenneth B. Crozier, Federico Capasso, David Bour, Scott Corzine, and Gloria Höfler. 2007. "Plasmonic Quantum Cascade Laser Antenna." Applied Physics Letters 91 (17): 173113. https://doi.org/10.1063/1.2801551.

\section{Permanent link}

http://nrs.harvard.edu/urn-3:HUL.InstRepos:41372464

\section{Terms of Use}

This article was downloaded from Harvard University's DASH repository, and is made available under the terms and conditions applicable to Other Posted Material, as set forth at http:// nrs.harvard.edu/urn-3:HUL.InstRepos:dash.current.terms-of-use\#LAA

\section{Share Your Story}

The Harvard community has made this article openly available.

Please share how this access benefits you. Submit a story.

\section{Accessibility}




\title{
Plasmonic quantum cascade laser antenna
}

\author{
Nanfang Yu, ${ }^{\text {a) }}$ Ertugrul Cubukcu, Laurent Diehl, Mikhail A. Belkin, \\ Kenneth B. Crozier, and Federico Capasso ${ }^{\text {b) }}$ \\ School of Engineering and Applied Sciences, Harvard University, Cambridge, Massachusetts 02138, USA \\ David Bour, ${ }^{\mathrm{c})}$ Scott Corzine, ${ }^{\mathrm{d})}$ and Gloria Höfler ${ }^{\mathrm{e})}$ \\ Agilent Laboratories, 3500 Deer Creek Road, Palo Alto, California 94304, USA
}

(Received 4 June 2007; accepted 24 August 2007; published online 23 October 2007)

\begin{abstract}
We report a plasmonic quantum cascade laser antenna that confines coherent midinfrared radiation well below the diffraction limit. The antenna was fabricated on the facet of a midinfrared quantum cascade laser and consists of a pair of gold nanorods separated by a gap. The antenna near field was characterized by an apertureless near-field scanning optical microscope; field confinement of about 100 and $70 \mathrm{~nm}$, limited by the gap size, was demonstrated at wavelengths of 7.0 and $5.3 \mu \mathrm{m}$, respectively. This device may find important applications in midinfrared subwavelength chemical and biological imaging and spectroscopy. () 2007 American Institute of Physics.
\end{abstract}

[DOI: $10.1063 / 1.2801551]$

A compact subwavelength coherent radiation source operating in the "fingerprint region"1 of the midinfrared (midir) spectrum is crucial for resolving nanometric surface features of a biological or chemical specimen. The resolution of Fourier transform infrared microscopes is diffraction limited to a couple of microns. Apertureless near-field scanning optical microscopes (a-NSOMs) have been demonstrated, which can substantially exceed the diffraction limit in the mid-ir spectral region. ${ }^{2}$ In this technique the apex of a metallic tip illuminated by an external mid-ir source acts as a subwavelength local scatterer that can be scanned over the sample surface. a-NSOMs have previously been implemented to perform high-resolution mid-ir near-field microscopy of polymers ${ }^{3,4}$ and viruses. ${ }^{5}$

Recently an active device, termed plasmonic laser antenna, capable of generating an intense nanometric $(\sim \lambda / 20)$ optical spot in the near field was demonstrated. ${ }^{6}$ It was implemented using commercial laser diodes emitting in the near infrared $(\lambda \approx 0.8 \mu \mathrm{m})$. In this letter, mid-ir plasmonic laser antennas, which provide a spatial field confinement below $100 \mathrm{~nm}$, are reported. These compact laser devices that do not require an external coherent illumination source and the intermediate optics can be utilized as active subwavelength probes for spatially resolved chemical and biological imaging and spectroscopy. ${ }^{3-5}$

Our devices were fabricated by defining on the facet of a quantum cascade laser (QCL) an optical antenna consisting of a pair of gold nanorods separated by a nanometric size gap. The antenna axis is orientated along the laser polarization. Resonance is achieved when the length of each nanorod approximately equals an odd integer number of half surface plasmon wavelengths. ${ }^{6-9}$ For a single rod antenna on resonance, the magnitude of the induced current in the rod is maximized, thereby maximizing the surface charge density at

\footnotetext{
${ }^{a)}$ Electronic mail: nyu@fas.harvard.edu

${ }^{b}$ Electronic mail: capasso@seas.harvard. edu

${ }^{c}$ Present address: Bridgelux Inc., 1225 Bordeaux Dr., Sunnyvale, California 94089, USA.

d) Present address: Infinera HQ, 169 Java Dr., Sunnyvale, California 94089, USA.

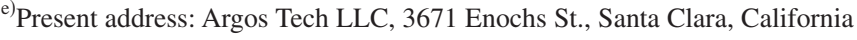
95051, USA.
}

the ends of the rods, leading to large values of the surface plasmon electric fields in their vicinity. ${ }^{7,8}$ Placing the two antenna rods in close proximity enhances the electric field in the gap due to capacitive coupling, since the rod ends that form the gap have charges of opposite sign. ${ }^{6}$ Resonances of passive mid-ir antennas have been observed in far field experiments. ${ }^{7,9}$ QCLs are semiconductor lasers based on optical transitions between quantum confined states in the conduction band. ${ }^{10,11}$ Mid-ir QCLs have recently achieved a high level of technological maturity providing continuous wave operation with hundreds of milliwatts of output power at and above room temperature with good reliability. ${ }^{12}$ The emission wavelength of QCLs can be adjusted continuously from about 3 to $24 \mu \mathrm{m}$ by tailoring the thickness of the active region quantum well layers, thus covering the entire mid-ir fingerprint region. ${ }^{1}$ A wide range of spectroscopybased chemical sensing and trace gas analysis techniques using QCLs have been demonstrated. ${ }^{13}$

To optimize the antenna design, finite-difference timedomain (FDTD) simulations were performed using a commercial software package (XFDTD, Remcom, Inc.). A schematic diagram of the simulated antenna geometry for a $\lambda=7.0 \mu \mathrm{m}$ QCL is shown in the inset of Fig. 1(a). In the simulations, gold is modeled as a modified Debye material. ${ }^{14}$ The mid-ir plane wave excitation polarized along the axis of the antenna was launched from the inside of the laser medium in the direction normal to the device facet, which is assumed to have dimensions much greater than the wavelength in the semiconductor (about $2.2 \mu \mathrm{m}$ at $\lambda=7.0 \mu \mathrm{m}$ ).

The simulation results are presented in Fig. 1 for a free space wavelength of $7.0 \mu \mathrm{m}$. The two peaks at $L=1.3$ and $5.1 \mu \mathrm{m}$ in Fig. 1(a) represent the first and second dipolar antenna resonances, respectively. ${ }^{7-9,15}$ As shown in Fig. 1(b), the near field of the resonant antenna is enhanced in the gap for an incident electric field polarized along the antenna length; simulations instead show negligible field enhancement in the gap when the incident electric field is perpendicular to the antenna length. The strong electric field for resonant excitation is confined to a region with a spatial extent mostly determined by the gap size rather than the excitation wavelength. We fabricated optical antennas on both $\lambda=7.0 \mu \mathrm{m}$ and $\lambda=5.3 \mu \mathrm{m}$ QCLs. As confirmed by simulations, a simple scaling of the resonant antenna design for the 

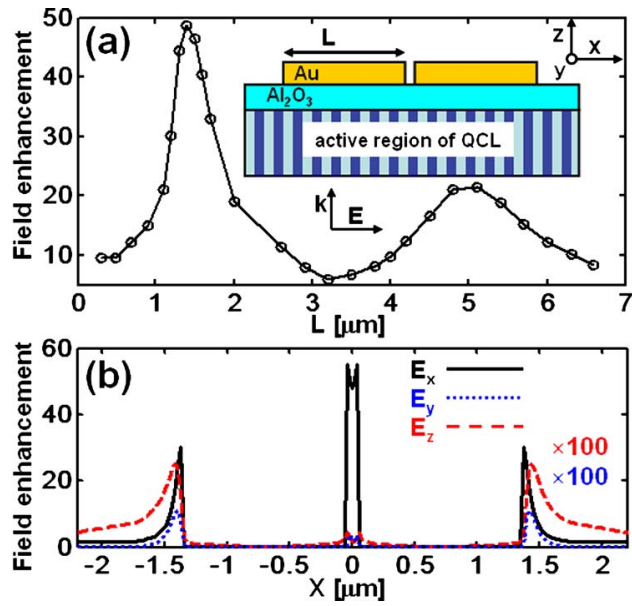

FIG. 1. (Color online) FDTD simulation results of mid-ir plasmonic antenna on a $\lambda=7.0 \mu \mathrm{m}$ quantum cascade laser (QCL). (a) Electric field amplitude enhancement vs the antenna length $L$. The field is calculated in the middle of the gap at the level of the antenna top surface and normalized to the amplitude of the incident field. Inset: schematic of the antenna structure. The antenna is defined on the cleaved facet of a QCL and oriented along the laser polarization, which is normal to the QCL quantum well layers schematically shown as vertical stripes. The antenna rod thickness and width are chosen to be 70 and $125 \mathrm{~nm}$, respectively, and the gap between the antenna rods is fixed at $100 \mathrm{~nm}$. The rod length $L$ is varied from 0.25 to $6.6 \mu \mathrm{m}$. The QCL material has a refractive index $n=3.15$ and the facet is assumed to be coated with a $70 \mathrm{~nm}$ thick electrically insulating layer of alumina. (b) Electric field amplitude enhancement at the level of the antenna top surface along the antenna axis for an antenna at the first resonance with $L=1.3 \mu \mathrm{m}$.

$\lambda=7.0 \mu \mathrm{m}$ QCLs by a factor of $5.3 / 7$ produces resonant antenna structures for the $\lambda=5.3 \mu \mathrm{m}$ QCLs.

The devices on which the antennas were fabricated are buried heterostructure (BH) QCLs emitting around $\lambda=5.3 \mu \mathrm{m}$ and $\lambda=7.0 \mu \mathrm{m}$. The BH processing and the performance of these structures are described in Refs. 12, 16, and 17. QCL spectra above threshold were measured to determine the lasing wavelength and the corresponding resonant antenna length. A mid-ir a-NSOM (Fig. 2) based on a commercial atomic force microscope (AFM) (PSIA XE-120

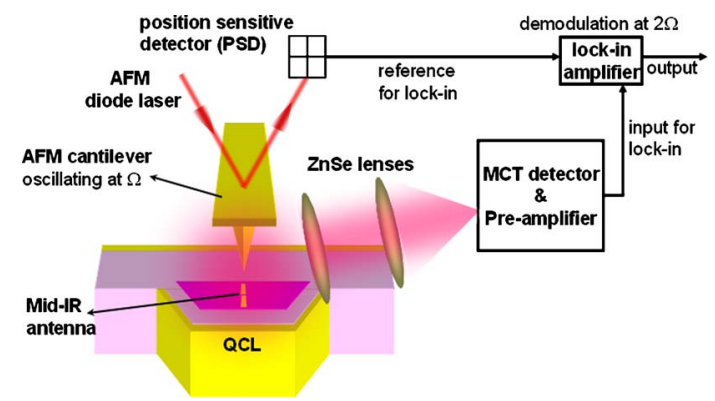

FIG. 2. (Color online) Midinfrared apertureless NSOM setup used in the experiments. For these measurements, commercial silicon AFM cantilevers (Veeco Probes) were used that had tip apex curvature of about $15 \mathrm{~nm}$. The tips were coated with $25 \mathrm{~nm} \mathrm{Au}$ and the tapping amplitude of the oscillating tips was about $30 \mathrm{~nm}$. The tilting of the tip axis in the experimental setup is about $20^{\circ}$ with respect to the laser facet normal. The quantum cascade lasers were operated in low duty cycle $(1 \%)$ pulsed mode to avoid overheating of the AFM tips or the antennas. The pulse repetition rate was hundreds of kilohertz, much higher than the tapping frequency of the AFM tips, typically about $40 \mathrm{kHz}$. The mid-ir radiation scattered by the AFM tip was collected and focused onto a nitrogen cooled mercury-cadmium-telluride (MCT) detector by two $f / 1.5$ zinc selenide ( $\mathrm{ZnSe}$ ) lenses. The collection angle was about $80^{\circ}$ from the normal to the laser facet. The signal from the MCT detector was fed into a lock-in amplifier (SRS830, Stanford Research Systems) that was set to demodulate at the second harmonic of the tapping

in the antenna gaps. Their size is comparable to the size of
fownloaded 24 Oct 2007 to 128.103.60.225. Redistribution subject to AIP license or copyright, see http://apl.aip.org/apl/copyright.jsp

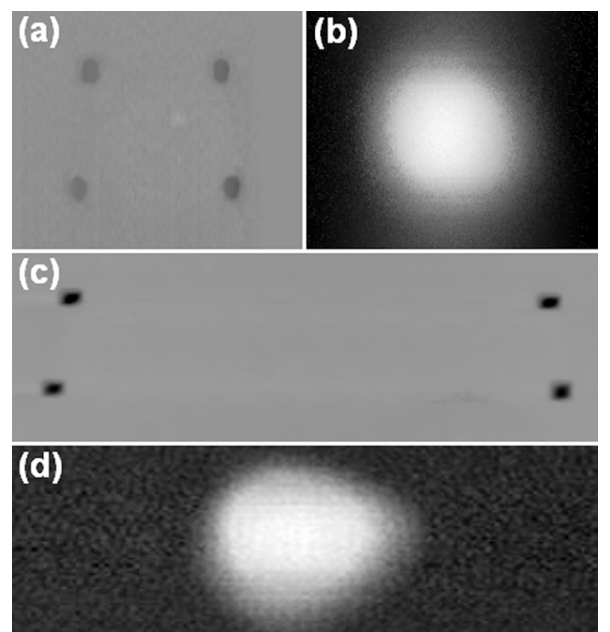

FIG. 3. Transverse mode characterization of quantum cascade lasers (QCLs). [(a) and (c)] AFM topography of the facets of a $\lambda=5.3 \mu \mathrm{m}$ QCL and a $\lambda=7.0 \mu \mathrm{m}$ QCL, respectively; the markers that can be observed in the topography images at the corners of the trapezoidal active regions are fabricated by focused ion beam milling and are used to help place the antenna at the peak of the optical mode. [(b) and (d)] NSOM images of the $\mathrm{TM}_{00}$ mode of the lasers shown in (a) and (c), respectively. Image size: [(a) and (b)] $8 \times 10 \mu \mathrm{m}^{2}$ and [(c) and (d)] $4.5 \times 14 \mu \mathrm{m}^{2}$.

or Park Scientific SPM CP-II) was then utilized to characterize the spatial mode profiles of the laser transverse modes for accurate positioning of the antennas on the mode maxima. Recently, this technique has been used to image the evanescent field of the Fabry-Pérot longitudinal modes on the socalled "air-waveguide" of a mid-ir QCL. ${ }^{18}$ We used lasers with relatively narrow active regions operating on a single $\left(\mathrm{TM}_{00}\right)$ transverse mode. Figure 3 shows typical laser mode imaging results for the two types of QCLs on which the antennas were defined: one had a $5 \mu \mathrm{m}$ wide active region and lased at $\lambda=5.3 \mu \mathrm{m}$ [Figs. 3(a) and 3(b)], the other had a $12.5 \mu \mathrm{m}$ wide active region and $\lambda=7.0 \mu \mathrm{m}$ [Figs. 3(c) and $3(\mathrm{~d})]$. The imaging of the modes was performed when the QCLs were operated about 50\% above the threshold current with an average power of about 0.4 and $1.1 \mathrm{~mW}$ for the $\lambda=5.3 \mu \mathrm{m}$ and $\lambda=7.0 \mu \mathrm{m}$ devices, respectively.

After the initial characterization steps, the insulating $\mathrm{Al}_{2} \mathrm{O}_{3}$ and the $\mathrm{Au}$ layers were deposited on the laser facet. Then, the antenna structures were defined at the position of the laser mode maxima by focused ion beam milling. The light output versus current characteristics of the devices were subsequently measured to ensure that the lasers were still functioning properly. The mid-ir a-NSOM (Fig. 2) was utilized to measure the near field of the antenna structures. Lock-in detection at the second harmonic of the tapping frequency of the AFM tip was utilized to discriminate the scattered near fields from the optical background that originated mainly from reflection and scattering of laser light from the tip shaft and the cantilever end. ${ }^{19,20}$

The topography and the near-field images of the antennas were simultaneously measured (Fig. 4). Figure 4(a) shows the AFM topography, the NSOM image, and the line scan of the NSOM image along the axis of a resonant antenna with $L=1.2 \mu \mathrm{m}$ defined on a $\lambda=7.0 \mu \mathrm{m}$ QCL. Similarly, Fig. 4(b) is for another resonant antenna with $L=0.76 \mu \mathrm{m}$ defined on a $\lambda=5.3 \mu \mathrm{m}$ QCL. The line scans of the two NSOM images for the resonant antennas show that sharp optical spots were achieved with dimensions $\leqslant 100 \mathrm{~nm}$ in the antenna gaps. Their size is comparable to the size of 

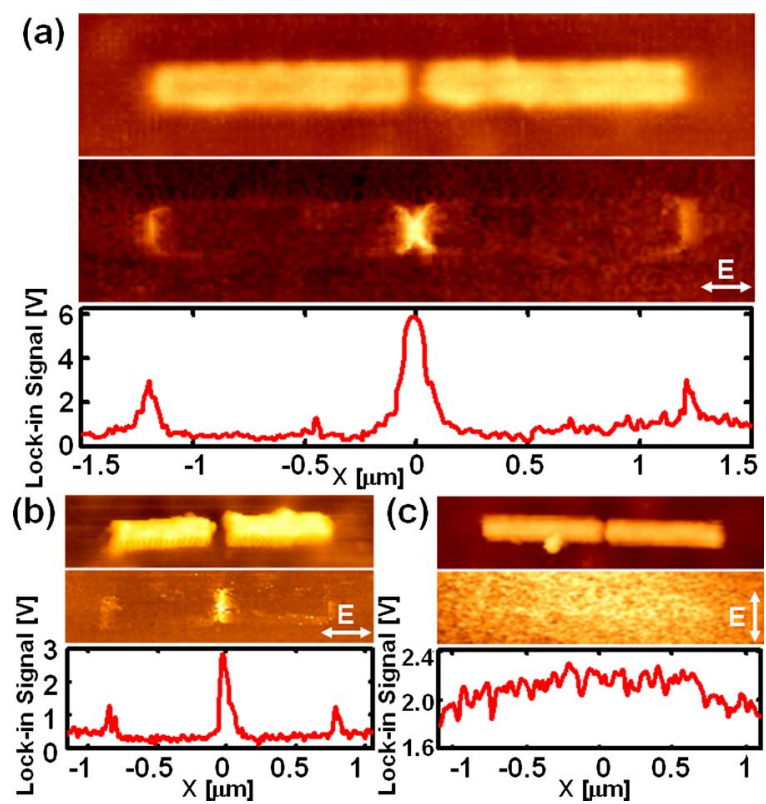

FIG. 4. (Color online) Mid-ir apertureless-NSOM imaging of plasmonic laser antennas. (a) Top and middle panels: simultaneous AFM topography and NSOM image for a resonant antenna with $L=1.2 \mu \mathrm{m}$ fabricated on the facet of a $\lambda=7.0 \mu \mathrm{m}$ QCL. Bottom panel: corresponding line scan along the antenna axis. (b) Top and middle panels: simultaneous AFM topography and NSOM image for a resonant antenna with $L=0.76 \mu \mathrm{m}$ fabricated on the facet of a $\lambda=5.3 \mu \mathrm{m}$ QCL. Bottom panel: corresponding line scan along the antenna axis. (c) Top and middle panels: simultaneous AFM topography and NSOM image of an antenna with $L=0.79 \mu \mathrm{m}$ fabricated on the facet of a $\lambda=5.3 \mu \mathrm{m}$ QCL but with orientation normal to the laser field. Bottom panel: corresponding line scan along the antenna axis. The polarization of the incident electric field is indicated in each NSOM image.

the antenna gaps, which are about 100 and $70 \mathrm{~nm}$ for the two in the antenna gap for the resonant structure using the estimated field strength in the QCL mode and the simulated field amplitude enhancement. For a $\lambda=7.0 \mu \mathrm{m}$ QCL working at an average power of $0.1 \mathrm{~mW}$ with $1 \%$ duty cycle, we obtained an optical intensity of about $0.5 \mathrm{GW} / \mathrm{cm}^{2}$ in the center of the gap, which corresponds to an electric field of about $3 \times 10^{5} \mathrm{~V} / \mathrm{cm}$. The field strength decreases to half of this value at about $50 \mathrm{~nm}$ above the antenna top surface. Figure 4(a) also shows that the resonant antenna at $\lambda=7.0 \mu \mathrm{m}$ has a ratio of the near field strength in the gap to the near field strength at antenna ends about 2, which is larger than that of an off-resonant antenna with $L=0.6 \mu \mathrm{m}$ at $\lambda=7.0 \mu \mathrm{m}$; the latter has a ratio of about 1.5 but the corresponding NSOM image is not shown. The physical reason is that at resonance the induced surface charges at the antenna ends are maximized so the capacitive coupling between two antenna rods in the resonant case is more efficient than that in the offresonant case. A bow-tie antenna structure may help eliminate the bright spots at antenna ends, ${ }^{6}$ which will make the device more suitable for applications. As a control experiment, Fig. 4(c) shows an antenna with $L=0.79 \mu \mathrm{m}$ at $\lambda$ $=5.3 \mu \mathrm{m}$. It has the same design as the one shown in Fig. 4(b) but with the antenna orientation perpendicular to the laser polarization. No noticeable field enhancement was observed [Fig. 4(c)].

The antenna near field images cannot be claimed to quantitatively represent the near field amplitude or intensity distribution because the measurements were not performed devices, respectively. We estimated the electric field strength interferometrically with a controlled reference beam as in

Refs. 3, 5, and 19. Therefore, the near-field intensity and the interference between the near field and the background fields coexist in the NSOM images, making it difficult to quantify the field enhancement. It should also be pointed out that the near-field scattering efficiency of the AFM tip is different when the field is polarized parallel or perpendicular to the axis of the AFM tip: the former has a much higher efficiency. Although the near-field is primarily polarized perpendicular to the AFM tip in our experimental configuration, there is still a nonzero field component along the tip axis due to the pyramidal shape of the tip, the tilting of the tip axis in the experimental setup and the abundant field components near the gap and at the ends of the antenna structure [Fig. 1(b)].

In conclusion, we have experimentally demonstrated mid-ir plasmonic laser antennas. The devices can provide an optical field confinement of $\lambda / 70$ at mid-ir wavelengths. The peak intensity in the gap of the resonant antenna is estimated to be on the order of $1 \mathrm{GW} / \mathrm{cm}^{2}$. These compact devices can add a dimension to high-resolution chemical and biological imaging and spectroscopy.

F. C. acknowledges support from the Air Force Office of Scientific Research (AFOSR MURI on Plasmonics) and the Harvard Nanoscale Science and Engineering Center (NSEC). K. C. acknowledges support from NSEC, Draper Laboratory and DARPA. Support from the Center for Nanoscale Systems (CNS) at Harvard University is also gratefully acknowledged. Harvard-CNS is a member of the National Nanotechnology Infrastructure Network (NNIN).

${ }^{1}$ J. W. Cooper, Spectroscopic Techniques for Organic Chemists (Wiley, New York, 1980), Chap. 1, p. 18.

${ }^{2}$ A. Lahrech, R. Bachelot, P. Gleyzes, and A. C. Boccara, Opt. Lett. 21, 1315 (1996).

${ }^{3}$ T. Taubner, R. Hillenbrand, and F. Keilmann, Appl. Phys. Lett. 85, 5064 (2004).

${ }^{4}$ M. B. Raschke, L. Molina, T. Elsaesser, D. H. Kim, W. Knoll, and K. Hinrichs, ChemPhysChem 6, 2197 (2005).

${ }^{5}$ M. Brehm, T. Taubner, R. Hillenbrand, and F. Keilmann, Nano Lett. 6, 1307 (2006)

${ }^{6}$ E. Cubukcu, E. A. Kort, K. B. Crozier, and F. Capasso, Appl. Phys. Lett. 89, 093120 (2006).

${ }^{7}$ K. B. Crozier, A. Sundaramurthy, G. S. Kino, and C. F. Quate, J. Appl. Phys. 94, 4632 (2003).

${ }^{8}$ R. W. P. King, H. R. Mimno, and A. H. Wing, Transmission Lines, Antennas and Wave Guides (McGraw-Hill, New York, 1945), Chap. II, Sec. 29, p. 160.

${ }^{9}$ F. Neubrech, T. Kolb, R. Lovrincic, G. Fahsold, A. Pucci, J. Aizpurua, T. W. Cornelius, M. E. Toimil-Molares, R. Neumann, and S. Karim, Appl. Phys. Lett. 89, 253104 (2006).

${ }^{10}$ J. Faist, F. Capasso, D. L. Sivco, C. Sirtori, A. L. Hutchinson, and A. Y. Cho, Science 264, 553 (1994).

${ }^{11}$ F. Capasso, C. Gmachl, D. L. Sivco, and A. Y. Cho, Phys. Today 55 (5), 34 (2002).

${ }^{12}$ L. Diehl, D. Bour, S. Corzine, J. Zhu, G. Höfler, M. Lončar, M. Troccoli, and F. Capasso, Appl. Phys. Lett. 88, 201115 (2006).

${ }^{13}$ A. A. Kosterev and F. K. Tittel, IEEE J. Quantum Electron. 38, 582 (2002).

${ }^{14}$ K. S. Kunz and R. J. Luebbers, The Finite Difference Time Domain Method for Electromagnetics (CRC, Boca Raton, FL, 1993), Chap. 8, p. 135.

${ }^{15}$ I. Codreanu and G. D. Boreman, Microwave Opt. Technol. Lett. 29, 381 (2001).

${ }^{16}$ M. Troccoli, S. Corzine, D. Bour, J. Zhu, O. Assayag, L. Diehl, B. G. Lee, G. Höfler, and F. Capasso, Electron. Lett. 41, 1059 (2005).

${ }^{17}$ L. Diehl, D. Bour, S. Corzine, J. Zhu, G. Höfler, B. G. Lee, C. Y. Wang, M. Troccoli, and F. Capasso, Appl. Phys. Lett. 88, 041102 (2006).

${ }^{18}$ V. Moreau, M. Bahriz, R. Colombelli, P. Lemoine, Y. D. Wilde, L. R. Wilson, and A. B. Krysa, Appl. Phys. Lett. 90, 201114 (2007).

${ }^{19}$ R. Hillenbrand, B. Knoll, and F. Keilmann, J. Microsc. 202, 77 (2001).

${ }^{20}$ B. Knoll and F. Keilmann, Opt. Commun. 182, 321 (2000). Downloaded 24 Oct 2007 to 128.103.60.225. Redistribution subject to AIP license or copyright, see http://apl.aip.org/apl/copyright.jsp 